\title{
Impact of Gravity on Vacuum Stability
}

\author{
Vincenzo Branchina ${ }^{1,2}$, Emanuele Messina ${ }^{1,2}$, and D. Zappalà ${ }^{2}$ \\ ${ }^{1}$ Department of Physics and Astronomy, University of Catania, Via Santa Sofia 64, 95123 Catania, Italy and \\ 2 INFN, Sezione di Catania, Via Santa Sofia 64, 95123 Catania, Italy
}

(Dated: August 13, 2018)

\begin{abstract}
In a pioneering paper on the role of gravity on false vacuum decay, Coleman and De Luccia showed that a strong gravitational field can stabilize the false vacuum, suppressing the formation of true vacuum bubbles. This result is obtained for the case when the energy density difference between the two vacua is small, the so called thin wall regime, but is considered of more general validity. Here we show that when this condition does not hold, however, a strong gravitational field (Planckian physics) does not necessarily induce a total suppression of true vacuum bubble nucleation. Contrary to common expectations then, gravitational physics at the Planck scale does not stabilize the false vacuum. These results are of crucial importance for the stability analysis of the electroweak vacuum and for searches of new physics beyond the Standard Model.
\end{abstract}

PACS numbers: 14.80.Bn, 11.27.+d, 04.62.+v

Introduction - The search for new physics beyond the Standard Model (BSM) is one of the most important and challenging questions of present experimental and theoretical physics. The first run of LHC did not show any sign of new physics [1]. The second run, reporting local excess of signal in the diphoton channel, has generated a certain expectation and enthusiasm in the community [2]. Whether or not this signal will be confirmed by future work, clues are also needed from the theoretical side. In this respect, crucial for our understanding and construction of BSM theories is the stability analysis of the electroweak (EW) vacuum [3-9]. The discovery of the Higgs boson boosted new interest on this question, making it one of the hottest topic in theoretical particle physics [10-17]. At first, it seemed that the knowledge of the Higgs and top masses, $M_{H}$ and $M_{t}$, was sufficient to determine the stability condition of the SM vacuum, that is to determine whether it is a metastable state (false vacuum) or a stable one (true vacuиm). It was later realized, however, that this knowledge is not enough, as the stability condition of the vacuum is very sensitive to unknown new physics interactions at high (or very high, say Planckian) energy scales [14-17].

Following a pioneering work of Bender and collaborators [18], Coleman and Callan studied the decay of the false vacuum in a flat space-time background [19]. Later, Coleman and De Luccia extended this analysis to include the impact of gravity [20]. The physical mechanism that triggers the false vacuum decay is quite simple: due to quantum fluctuations, there is a finite probability that a bubble of true vacuum materializes in the sea of false vacuum.

Coleman and collaborators worked within the so called "thin wall" approximation, that applies when the two minima of the potential, $\phi_{\text {false }}$ and $\phi_{\text {true }}$, are such that the energy density difference $V\left(\phi_{\text {false }}\right)-V\left(\phi_{\text {true }}\right)$ is much smaller than the height of the potential barrier $V\left(\phi_{t o p}\right)-V\left(\phi_{\text {false }}\right)$, where $V\left(\phi_{t o p}\right)$ is the maximum of the potential between the two minima at $\phi_{\text {false }}$ and $\phi_{\text {true }}$. They found that the probability of materialization of a true vacuum bubble decreases for increasing values of its size. In a flat space-time background, this probability turns out to be always finite, no matter how large the bubble size. In a curved background, things are different. In the following we concentrate on the case of a false vacuum with vanishing energy density, Minkowski vacuum, and a true vacuum with negative energy density, anti-de Sitter (AdS) vacuum, as this case is very relevant for applications, in particular for the stability analysis of the EW vacuum.

The transition from a false Minkowski vacuum to a true AdS vacuum was studied in [20], where it was shown that, when the size of the Schwarzschild radius of the bubble of true vacuum is much smaller than its size, i.e. when the gravitational effects are weak, the probability of materialization of such a bubble is close to the flat space-time result, while, when the Schwarzschild radius becomes comparable with the bubble size, i.e. in a strong gravitational regime, the presence of gravity stabilizes the false vacuum, preventing the materialization of a true vacuum bubble. This result was obtained under the thin wall condition mentioned above, namely for the case when $V\left(\phi_{\text {false }}\right)-V\left(\phi_{\text {true }}\right)<<V\left(\phi_{\text {top }}\right)-V\left(\phi_{\text {false }}\right)$. However, it is commonly considered as being of more general validity.

Going back to the SM, it is known that due to the top loop corrections the Higgs potential $V(\phi)$ bends down for values of $\phi>\phi_{\min }^{(1)}=v$, where $v \sim 246 \mathrm{GeV}$ is the location of the EW minimum, and for the present experimental values of $M_{H}$ and $M_{t}$, namely $M_{H} \sim 125.09 \mathrm{GeV}$ and $M_{t} \sim 173.34 \mathrm{GeV}$ [21, 22], develops a second minimum, much deeper than the EW one and at a much larger value of the field, $\phi_{\min }^{(2)}>>v$. Clearly, the conditions under which the Coleman-De Luccia result is derived are not fulfilled. As said above, however, it is still expected that in the presence of Planckian physics the bubble nucleation rate vanishes [23-25]. In more technical words, it is expected that the bounce solution to the euclidean equation of motion (from which the saddle point approximation for the bubble nucleation rate is obtained) disappears.

Motivated by the above phenomenological considerations, in this Letter we focus our attention on the case that is relevant for the applications to the analysis of the SM vacuum decay, namely the decay of a false vacuum with zero energy density to a true vacuum with negative energy density, i.e. the decay 


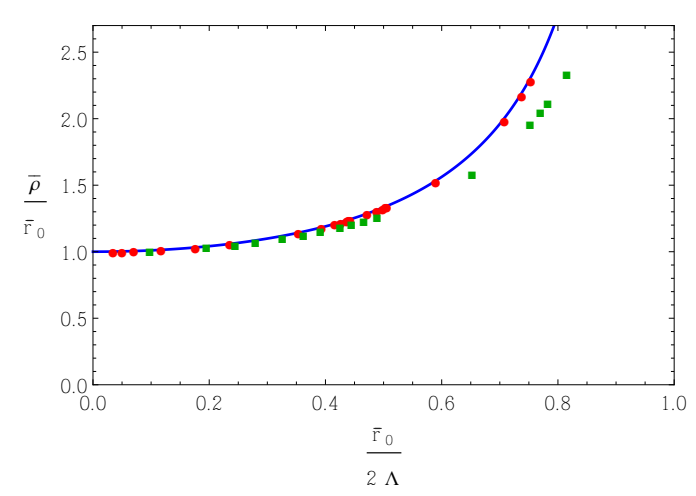

FIG. 1: $\bar{\rho} / \bar{r}_{0}$ computed for $b=0.01$ (red online) circles, and for $b=0.13$ (green online) squares, plotted together with the thin wall prediction, (blue online) solid line.

from a Minkowski false vacuum state to an AdS true vacuum. To study the impact of gravity on the nucleation rate of a bubble of true vacuum, we consider a potential [26] that allows to explore in a unified framework all possible cases, from the thin wall regime to the case when the difference in height between the two minima of the potential is large. For this latter case, we find that the nucleation rate of a true vacuum bubble has a value close to the result obtained in flat space-time even in a strong gravity regime, when the typical mass scales of the potential (see Eq. (2) below) become comparable with (or even larger than) the Planck scale.

In fact, we will see that in this case no vanishing of the bubble materialization rate is observed at these high energy scales. On the contrary, we find that the suppression of the Minkowski false vacuum decay (if still present) is pushed to very high energy regimes, even much higher than the Planck scale, so that the possible presence of a Coleman-De Luccia pole becomes physically irrelevant (similarly to what happens for the Landau pole in QED, where the latter occurs at such a high energy scale that the theory has already lost its significance several orders of magnitudes below that scale). At energies below the pole scale, but yet Planckian or transPlanckian, the decay probability is non-vanishing and very close to the flat space-time result, i.e. the result obtained ignoring the presence of gravity.

These findings are new and totally unexpected, as it was thought that in a strong gravity regime the decay of a false Minkoski vacuum to a true AdS vacuum is always inhibited. Moreover, they are of crucial importance for current studies and for model building of BSM physics, where we are often interested in considering new physics at Planckian and/or transPlanckian scales.

Analysis - To study the impact of gravity on false vacuum decay, let us consider the (Euclidean) action of a scalar field in curved space-time together with the Einstein term:

$$
S=\int d^{4} x \sqrt{g}\left[\frac{1}{2} g^{\mu v} \partial_{\mu} \phi \partial_{v} \phi+V(\phi)-\frac{R}{16 \pi G}\right],
$$

where $R$ is the Ricci scalar, $G$ the Newton constant, and $V(\phi)$

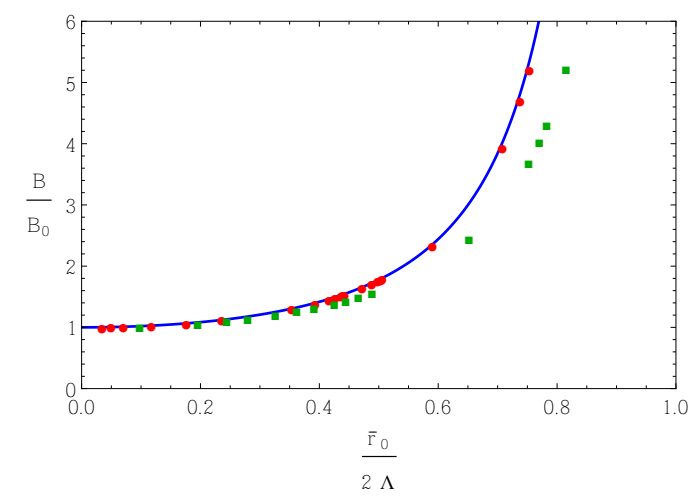

FIG. 2: $B / B_{0}$ computed for the same values of the parameters used in Fig.1.

the potential [26]:

$$
V(\phi)=\frac{g^{2}}{4}\left[\left(\phi^{2}-a^{2}\right)^{2}+\frac{4 b}{3}\left(a \phi^{3}-3 a^{3} \phi-2 a^{4}\right)\right] .
$$

When $0<b<1, V(\phi)$ has two non-degenerate minima at $\phi= \pm a, \phi=a$ being the absolute minimum, and a potential barrier that separates the two minima, with maximum at $\phi=-b a$. Note that for $b=0$ this is just the double well potential with two degenerate minima at $\phi= \pm a$, while for $b=1$ the minimum at $\phi=-a$ becomes an inflection point and the barrier disappears.

This potential is perfectly suited for our scopes. By varying the dimensionless parameter $b$, we control the difference in height, $V(-a)-V(a)$, between the false and the true vacuum. The comparison between this difference and the height of the potential barrier, $V(-b a)-V(-a)$, allows to determine whether or not we are in the thin wall regime, the latter occuring when $V(-a)-V(a)<<V(-b a)-V(-a)$. At the same time, by varying the dimensionful parameter $a$ (the only mass scale of our potential), and pushing it toward the Planck scale and beyond, we will be able to test the impact of strong gravitational physics on the decay of the false vacuum.

Anticipating from the following analysis, we will see that in the $b \rightarrow 1$ limit (that is the limit of the largest possible difference $V(-a)-V(a)$ for the potential (2)), the decay probability from the Minkowski false vacuum to the true vacuum gives exactly the flat space-time result (as if gravity was absent!), for any value of $a$, i.e. from very weak to very strong gravitational regimes. For values of $b$ close to (but smaller than) 1 , the suppression of the vacuum decay should occur for very large transplanckian values of $a$.

To calculate the bubble nucleation rate $\Gamma$ we need the bounce solution to the (Euclidean) equations of motion derived by varying the action in Eq. (1). The saddle point approximation to the transition rate $\Gamma$ per unit volume is given by the ratio between the exponential of minus the (Euclidean) action evaluated at the bounce and the action calculated for the false vacuum solution [20]:

$$
\frac{\Gamma}{\mathscr{V}} \sim e^{-\left(S_{\text {bounce }}-S_{\text {false }}\right)} \equiv e^{-B}
$$




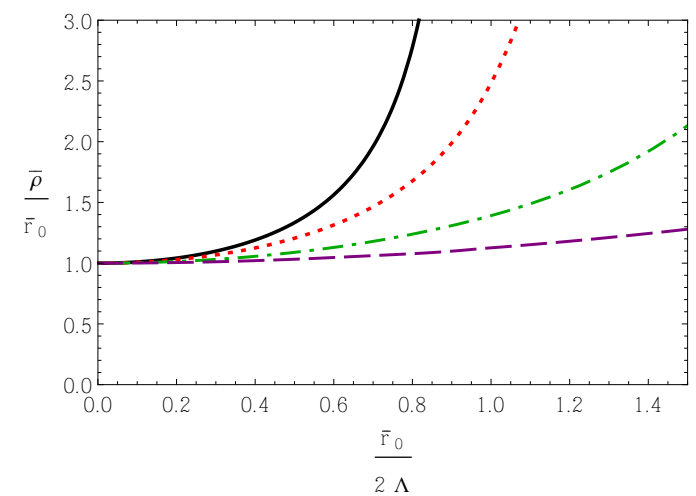

FIG. 3: $\bar{\rho} / \bar{r}_{0}$ computed for $b=0.4$ (red online) dotted, $b=0.7$ (green online) dot-dashed, $b=0.85$ (violet online) dashed line. The black solid curve corresponds to the thin wall prediction.

Following [20], we consider the most general $O(4)$ symmetric Euclidean metric ( $r$ is the radial coordinate along a radial curve):

$$
(d s)^{2}=(d r)^{2}+\rho(r)^{2}\left(d \Omega_{3}\right)^{2}
$$

where $d \Omega_{3}$ is the element of distance on a unit three-sphere and $\rho(r)$ the radius of curvature of each three-sphere.

With the metric (4), the Einstein equation $G_{r r}=-\kappa T_{r r}$ reduces to the following equation for $\rho(r)$ (the ' denotes derivation with respect to $r$ ):

$$
\rho^{\prime 2}=1+\frac{\kappa}{3} \rho^{2}\left(\frac{1}{2} \phi^{\prime 2}-V(\phi)\right)
$$

where $\kappa \equiv 8 \pi G \equiv 8 \pi / M_{P}^{2}$, and $M_{P}$ is the Planck scale. Eq. [5] is coupled to the scalar field equation derived from (1),

$$
\phi^{\prime \prime}+\frac{3 \rho^{\prime}}{\rho} \phi^{\prime}=\frac{d V}{d \phi} .
$$

The false vacuum solution to these coupled equations consists of the flat space-time metric, $\rho_{\text {false }}^{\prime}(r)=1$, together with the constant solution for the profile, $\phi_{\text {false }}(r)=-a$.

In order to compute $\Gamma$, we have to select the classical solutions of Eqs. (5) and (6) that fulfill the boundary conditions $\phi(+\infty)=-a, \phi^{\prime}(0)=0$ and $\rho(0)=0$. In our case, the action in Eq. (1) can be written as:

$$
\begin{aligned}
S=\quad & 2 \pi^{2} \int_{0}^{\infty} d r\left[\rho^{3}\left(\frac{1}{2} \phi^{\prime 2}+V(\phi)\right)+\right. \\
& \left.\frac{3}{\kappa}\left(\rho^{2} \rho^{\prime \prime}+\rho \rho^{\prime 2}-\rho\right)\right]= \\
& 4 \pi^{2} \int_{0}^{\infty} d r\left(\rho^{3} V(\phi)-\frac{3 \rho}{\kappa}\right)+B . T .
\end{aligned}
$$

where the right hand side is obtained by making use of Eqs. (5) and (6), and the boundary terms B.T. appear after integrating by parts $\rho^{2} \rho^{\prime \prime}$ in the second line of Eq. 7 .

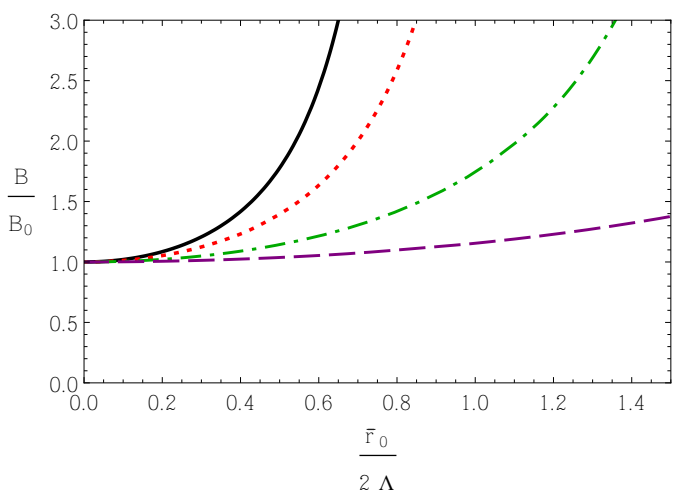

FIG. $4: B / B_{0}$ with the same coding as in Fig. 3 .

The B.T. are infinite. However, the computation of $\Gamma$ only involves the difference $B$ between the action evaluated at the bounce and at the false vacuum solutions (see Eq. (3)). These two solutions coincide at infinity, and the two actions provide the same divergent contribution, that is then canceled out in the difference $B=S_{\text {bounce }}-S_{\text {false }}$.

It is worth noting that, due to the particular dependence on the coupling $g$ of the potential $V(\phi)$ in (2), it is possible to re-express Eqs. (5) and (6) in terms of the rescaled quantities $\hat{r}=g r, \hat{\rho}=g \rho$ and $\hat{V}=V / g^{2}$, so that no explicit dependence on $g$ is left. Accordingly, the dependence on $g$ of the action can be factored out: $S=\hat{S} / g^{2}$ and $B=\hat{B} / g^{2}$.

To calculate the transition rate $\Gamma$ for different potentials of the kind given in Eq. (2), i.e. for different values of the parameters $a$ and $b$, we need first to solve the system of coupled differential equations (5) and (6) with the boundary conditions given above.

We do that with the help of a numerical shooting procedure. The typical bounces are decreasing profiles $\phi_{b}(r)$, such that $\phi_{b}(r \rightarrow \infty) \rightarrow-a$, and can be parametrized by their size $\bar{r}$, the value of the radial coordinate where the height of the profile is decreased of one half. For large values of $r$, i.e. for $r>>\bar{r}$, the solution for the radius of curvature $\rho(r)$ approaches the flat space-time solution, $\rho^{\prime}(r)=1$, while for $r<<\bar{r}$, i.e. inside the bubble of true vacuum, the presence of a negative energy density creates a distortion of this solution.

By defining $\bar{\rho}$ as the value of the curvature at $r=\bar{r}$, that is $\bar{\rho} \equiv \rho(\bar{r})$, a simple way of displaying our findings is to compare the results obtained for $B$ and $\bar{\rho}$ with the corresponding quantities derived in the flat space-time case, that will be indicated as $B_{0}$ and $\bar{r}_{0}$ respectively (needless to say, in this latter case $\rho(r)$ coincides with $r$ ).

In the thin wall regime, that for our potential is the case of small values of the parameter $b$, it was shown in [20] that:

$\bar{\rho}=\bar{r}_{0}\left[1-\left(\bar{r}_{0} /(2 \Lambda)\right)^{2}\right]^{-1}$ and $B=B_{0}\left[1-\left(\bar{r}_{0} /(2 \Lambda)\right)^{2}\right]^{-2}$, i.e. $\bar{\rho}$ and $B$ have a pole at $\bar{r}_{0} / 2=\Lambda \equiv \sqrt{3 /(8 \pi G|V(a)|}$. The corresponding curves are reported in Figs.1, 2, 3, 4 as solid lines having an asymptote at $\bar{r}_{0} /(2 \Lambda)=1$.

Our numerical approach allows to check the accuracy of the thin wall predictions, and before moving to the cases of partic- 
ular interest to us, we calculate $\bar{\rho} / \bar{r}_{0}$ and $B / B_{0}$ for some small values of $b$ (thin wall regime). To facilitate the comparison with [20], we present our results by plotting $\bar{\rho} / \bar{r}_{0}$ and $B / B_{0}$ against $\bar{r}_{0} /(2 \Lambda)$, that is an increasing function of $a$ for each fixed value of $b$. The parameter $a$ gives the location of the minima, and increasing values of $a$ toward the Planck scale (and beyond) mark the transition from the weak to the strong gravitational regime. The results are plotted in Figs.11and 2 . Note that the rescaling to the hatted quantities discussed above shows that all variables on the $x$ and $y$ axes of the figures do not depend on the coupling $g$.

For the smallest value considered for $b, b=0.001$, the points practically sit on the thin wall curve (blue online solid curve), and are not reported in Figs. 1 and 2. At $b=0.01$ (red online circles) we start to observe a small deviation from the thin wall curve, that becomes more sizable for $b=0.13$ (green online squares). Figs. 1 and 2 show that for small values of $b$, when the two minima of the potential are almost degenerate (thin wall regime), starting from $\bar{r}_{0} /(2 \Lambda) \sim 0.5$ the effect of gravity grows rapidly for increasing values of $\bar{r}_{0} /(2 \Lambda)$.

We now consider larger values of $b$, thus leaving the thin wall regime. Some of the curves for $\bar{\rho} / \bar{r}_{0}$ and $B / B_{0}$ versus $\bar{r}_{0} /(2 \Lambda)$ resulting from our numerical analysis, namely those obtained for $b=0.4,0.7,0.85$, are reported in Figs. 3 and 4 . These figures clearly show that for increasing values of $b$, i.e. when the difference in height between the two minima increases, both curves $B / B_{0}$ and $\bar{\rho} / \bar{r}_{0}$ get flattened.

Moreover, for larger and larger values of $b(b \rightarrow 1)$, both curves get closer and closer to the orizontal lines $B / B_{0}=1$ and $\bar{\rho} / \bar{r}_{0}=1$. We have not added other curves in Figs. 3 and 4 as they would unnecessarily clutter these figures. For the same reason, we have also limited the range of values of $\bar{r}_{0} /(2 \Lambda)$ to $0<\bar{r}_{0} /(2 \Lambda)<1.5$. In fact, the Coleman-De Luccia pole (of the thin wall case) occurs at $\bar{r}_{0} /(2 \Lambda)=1$, and Figs. 3 and 4 clearly show that this point is not a pole for large values of $b$.

Finally, for $b \rightarrow 1$ these curves reach the horizontal lines $B / B_{0}=1$ and $\bar{\rho} / \bar{r}_{0}=1$ for the whole range of values $0<$ $\bar{r}_{0} /(2 \Lambda)<\infty$. This latter result is immediately found by considering the bounce profile function $\phi_{b}(r)$, that connects the center of the bounce, $\phi_{b}(0)$, with the false vacuum state at $r \rightarrow \infty, \phi_{b}(\infty)=-a$, and seeking for the numerical solution of Eq. 6). For small values of $b, \phi_{b}(0)$ is close to $a$, the true vacuum, while for increasing values of $b, \phi_{b}(0)$ decreases toward $\phi_{b}(0) \sim-a$. In the limit $b \rightarrow 1, \phi_{b}(0)$ reaches this latter value, so that

$$
\lim _{b \rightarrow 1} \phi_{b}(r)=-a
$$

in the whole range of $r$. In this limit, Eq. (5) becomes $\rho^{\prime}(r)=$ 0 , and we recover the flat space-time result.

These are the central results of the present Letter. In fact, the lesson from [20] (see Figs.11 and 2] is that when we move from weak to strong gravity regime, the impact of gravity becomes enormous, to the point that, for the critical value $\bar{r}_{0} /(2 \Lambda)=1, B$ diverges and the corresponding rate $\Gamma$ for the materialization of a bubble of true vacuum vanishes. As stressed above, this is expected to hold even out of the thin wall regime, and models of BSM physics and conclusions on the vacuum stability analysis are often based on this expectation $[23-25]$.

However, we have seen that, when the energy density difference between the two vacua of the potential increases (so that the conditions of [20] are no longer verified), the gravitational contribution to the transition rate $\Gamma$ becomes smaller and smaller. Moreover, when this difference becomes sufficiently large, that for our model occurs for $b \rightarrow 1$, the quencing of the vacuum decay does not occur even in very strong gravity regimes!

As mentioned before, these results are very surprising and unexpected, and also very important for phenomenological applications. In fact, extrapolating from [20], it was thought that whenever we enter in a strong gravity regime, i.e. when we approach (and possibly go beyond) the Planck scale, there should always be a strong suppression and even a vanishing of the false vacuum decay probability, resulting in a stabilization of the false vacuum. On the contrary, we see that for potentials where the difference in the depth of the minima is sufficiently large, this suppression (if still present) is pushed to very high regimes, even (much) above the Planck scale, so that the presence of a pole of $B / B_{0}$ becomes physically irrelevant ${ }^{1}$.

Moreover, the fact that in these conditions the transition rate from the false to the true vacuum is so close to the flat spacetime result is of crucial importance for phenomenological applications. In fact, the case that we have studied is relevant for the stability analysis of the electroweak vacuum, also in connection with present searches for BSM physics, where a stability analysis and a clear understanding of the role played by gravity is greatly needed.

\begin{tabular}{|c|c|c|}
\hline$b$ & $g \bar{r}_{0} a$ & $g^{2} B_{0}$ \\
\hline \hline 0.001 & 2121.32 & $4.44132 \times 10^{10}$ \\
\hline 0.01 & 212.113 & $4.44094 \times 10^{7}$ \\
\hline 0.13 & 16.2379 & 19921.0 \\
\hline 0.4 & 3.93098 & 276.288 \\
\hline 0.7 & 2.73633 & 70.9009 \\
\hline 0.85 & 2.51328 & 22.2602 \\
\hline
\end{tabular}

TABLE I: Set of values of $g \bar{r}_{0} a$ and $g^{2} B_{0}$ computed at different $b$.

For the reader's convenience, in Table I some values of $g \bar{r}_{0} a$ and $g^{2} B_{0}$ for different values of $b$ are reported. These are useful parameters to reconstruct physical quantities relevat to the analysis that we have presented and to better appreciate the results reported in the figures (see below). These quantities are obtained in the flat space-time case, and are both $g$-independent (as can be seen by resorting to the hatted vari-

\footnotetext{
${ }^{1}$ In 27] arguments are given in favour of the existence of such a pole. However, in the cases of interest considered in the present paper, this pole would be in a region where the theory is no longer valid.
} 
ables introduced before) and $a$-independent (they are dimensionless quantities, and in the absence of gravity that brings in the dimensionful Newton constant $G$, they cannot depend on the only dimensionful variable $a$ ). Therefore, they are univocally determined once $b$ is given.

In particular, the values of $g \bar{r}_{0} a$ in Table $\mathbb{I}$ are useful to establish the relation between $\bar{r}_{0} /(2 \Lambda)$, that appears in the $x$ axis of all figures, and the ratio $a / M_{P}$ (where $M_{P}$ is the Planck mass) for each value of $b$, that is for each single curve in the figures. In fact, by recalling the definition of $\Lambda$, one obtains the following relation $\bar{r}_{0} /(2 \Lambda)=(2 / 3) \sqrt{2 \pi b}\left(g \bar{r}_{0} a\right)\left(a / M_{P}\right)$. Then, for instance, the point $\bar{r}_{0} /(2 \Lambda)=1$ corresponds for $b=$ 0.001 to $a \sim M_{P} / 100$, and for $b=0.85$ to $a \sim M_{P} / 4$ (i.e. we are already in a srong gravity regime).

If we now look at the curves in Fig.4, these examples clearly illustrate the central results of our work. In fact, we see that for increasing values of $b$, at fixed values of $\bar{r}_{0} /(2 \Lambda)$ (e.g. $\bar{r}_{0} /(2 \Lambda)=1$ ), the suppression of the gravitational effects is larger when the strong gravity Planckian regime $\left(a \rightarrow M_{P}\right)$ is approached. Proceeding to consider higher values of $b$ (not displayed in the figure), the action $B$, and then the decay rate of the false vacuum, stays closer and closer to the flat spacetime result for larger and larger values of $a$ (see the discussion above in relation with Eq.(8)), even for $a>>M_{P}$ ! Finally, with the help of $g \bar{r}_{0} a$ and $g^{2} B_{0}$ in TableI] one can derive the values of $\bar{\rho}$ and $B$ from the various curves in the figures.

Before ending this Letter, we have to say some words on previous work [28] on the role of gravity on the EW vacuum decay, where a perturbative expansion of $\phi_{b}(r)$ and $\rho(r)$ in powers of $\kappa$ around the flat space-time solution was attempted. Actually, this analytical method seems to have a serious drawback, as the boundary conditions (see above), essential for the classical solution to be a bounce and in turn for $\Gamma$ in Eq. (3) to be the bubble nucleation rate, are not respected already at first order in $\kappa$. Therefore, the output of this analysis cannot be trusted and a fortiori cannot be used for comparison. A detailed study of this issue goes beyond the scope of the present paper and will be presented elsewhere.

Conclusions - We have studied the decay of a false Minkowski vacuum to a true AdS vacuum by taking into account the impact of gravity.

When the energy density difference between the false and the true vacuum is large compared to the height of the potential barrier (a condition that in the model discussed in the present Letter is obtained for $b \rightarrow 1$ in the potential of Eq. (2)), we find that the effect of gravity on the bubble production rate practically disappears even in the strong gravity regime, and the flat space-time result is recovered.

From a phenomenological point of view, the case that we have studied is of particular interest, as it is close to what happens in the stability analysis of the electroweak vacuum, and several models considered for physics beyond the Standard Model require a stability analysis where the role played by gravity needs to be carefully understood.

Therefore, the new results presented in this work are of the greatest importance for present studies on the SM stability problem and for searches of new physics beyond the Standard Model. They represent a real progress in understanding the impact of gravity on the stability of the vacuum. In the past, despite attempts to study this question [28], the role of gravity beyond the thin wall case was poorly understood.

[1] J. Olsen, CMS physics results from Run 2 presented on Dec. 15th, 2015, https://indico.cern.ch/event/442432/; CMS Collaboration, CMS PAS EXO-15-004, https://cds.cern.ch/record/ 2114808/files/EXO-15-004-pas.pdf.

[2] M. Kado, ATLAS physics results from Run 2 presented on Dec. 15th, 2015, https://indico.cern.ch/event/442432/; ATLAS Collaboration, ATLAS-CONF-2015-081, https://atlas.web.cern.ch/ Atlas/GROUPS/PHYSICS/CONFNOTES/ATLAS-CONF2015-081/.

[3] N. Cabibbo, L. Maiani, G. Parisi, R. Petronzio, Nucl. Phys. B158 (1979) 295.

[4] R.A. Flores, M. Sher, Phys. Rev. D27 (1983) 1679; M. Lindner, Z. Phys. 31 (1986) 295; M. Sher, Phys. Rep. 179 (1989) 273; M. Lindner, M. Sher, H. W. Zaglauer, Phys. Lett. B228 (1989) 139.

[5] C. Ford, D.R.T. Jones, P.W. Stephenson, M.B. Einhorn, Nucl. Phys. B395 (1993) 17.

[6] M. Sher, Phys. Lett. B317 (1993) 159.

[7] G. Altarelli, G. Isidori, Phys. Lett. B337 (1994) 141.

[8] J.A. Casas, J.R. Espinosa, M. Quirós, Phys. Lett. B342 (1995) 171; Phys. Lett. B382 (1996) 374.

[9] G. Isidori, G. Ridolfi, A. Strumia, Nucl. Phys. B609 (2001) 387.

[10] L.N. Mihaila, J. Salomon and M. Steinhauser, Phys. Rev. Lett. 108 (2012) 151602; K. Chetyrkin and M. Zoller, JHEP 06 (2012) 033; F. Bezrukov, M. Yu. Kalmykov, B. A. Kniehl, M. Shaposhnikov, JHEP 1210 (2012) 140.

[11] J. Elias-Miro, J.R. Espinosa, G.F. Giudice, G. Isidori, A. Riotto, A. Strumia, Phys. Lett. B709 (2012) 222.

[12] G. Degrassi, S. Di Vita, J. Elias-Miro, J.R. Espinosa, G.F. Giudice, G. Isidori, A. Strumia, JHEP 1208 (2012) 098.

[13] D. Buttazzo, G. Degrassi, P. P. Giardino, G. F. Giudice, F. Sala, A. Salvio, A. Strumia, JHEP 1312 (2013) 089.

[14] V. Branchina, E. Messina, Phys. Rev. Lett. 111 (2013) 241801.

[15] V. Branchina, E. Messina, A. Platania, JHEP 1409 (2014) 182.

[16] V. Branchina, E. Messina, M. Sher, Phys. Rev. D91 (2015) 013003.

[17] V. Branchina, E. Messina, "Stability and UV completion of the Standard Model", arXiv:1507.08812

[18] T. Banks, C. M. Bender, T. T. Wu, Phys.Rev. D8 (1973) 3346; Phys.Rev. D8 (1973) 3366.

[19] S. Coleman, Phys. Rev. D15 (1977) 2929; C. G. Callan, S. Coleman, Phys. Rev. D16 (1977) 1762.

[20] S. Coleman, F. De Luccia, Phys. Rev. D21 (1980) 3305.

[21] G. Aad et al. (ATLAS Collaboration, CMS Collaboration), Phys. Rev. Lett. 114 (2015) 19.

[22] ATLAS and CDF and CMS and D0 Collaborations, "First combination of Tevatron and LHC measurements of the top-quark mass", arXiv:1403.4427 [hep-ex].

[23] J. R. Espinosa, J.F. Fortin, M. Trépanier, "Consistency of Scalar Potentials from Quantum de Sitter Space", arXiv:1508.05343.

[24] L. Di Luzio, G. Isidori, G. Ridolfi, Phys.Lett. B753 (2016) 150.

[25] J. R. Espinosa, "Implications of the top (and Higgs) mass for vacuum stability", arXiv:1512.01222

[26] E. J. Weinberg, "Classical solutions in Quantum Field Theory", 
Cambridge University Press (2012).

[27] A. Aguirre, T. Banks, M. Johnson, JHEP 0608 (2006) 065. arXiv:hep-th/0603107.
[28] G. Isidori, V. S. Rychkov, A. Strumia, N. Tetradis, Phys.Rev. D77 (2008) 025034 